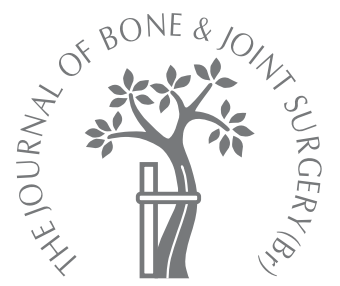

M. Khatri,

A. N. Stirrat

From Sunderland

Royal Hospital, Sunderland, England

M. Khatri, MS(Orth) FRCS(Tr \& Orth), Specialist Registrar (Orth)

A. N. Stirrat, FRCS(Ed) Consultant Orthopaedic Surgeon

Department of Orthopaedic Surgery

Sunderland Royal Hospital Kayll Road, Sunderland SR4 7TP, UK

Correspondence should be sent to Mr M. Khatri at 21 Angram Drive, Sunderland SR2 7RD, UK; e-mail: mkhatri9@hotmail.com

(C)2005 British Editorial Society of Bone and Joint Surgery doi:10.1302/0301-620X.87B7. $15588 \$ 2.00$

$J$ Bone Joint Surg [Br] 2005;87-B:950-4.

Received 20 April 2004;

Accepted after revision 10 November 2004

\title{
Souter-Strathclyde total elbow arthroplasty in rheumatoid arthritis
}

\author{
MEDIUM-TERM RESULTS
}

We present the outcome of 47 Souter-Strathclyde replacements of the elbow with a mean follow-up of 82 months (12 to 129). The clinical results were assessed using a conditionspecific outcome measure. The mean total score (maximum 100) before the operation was 47.21 and improved to 79.92 ( $p<0.001$ ). The mean pain score (maximum 50 ) improved from 21.41 to 46.70 ( $p<0.001$ ) and the mean functional component of the score (maximum 30 ) from 11.19 to 18.65 ( $p<0.001$ ). There was negligible change in the score for the range of movement although a significant improvement in mean flexion from $124^{\circ}$ to $136^{\circ}$ was noted (p $<0.001)$.

Revision surgery was required in four patients, for dislocation, wound dehiscence and early infection in one, late infection in two and aseptic loosening in one. The cumulative survival was $75 \%$ at nine years for all causes of failure and $97 \%$ at ten years for aseptic loosening alone. Our study demonstrates the value of the Souter-Strathclyde total elbow arthroplasty in providing relief from pain and functional improvement in rheumatoid patients.

In 1989 Souter $^{1}$ reported that the elbow was involved in approximately $50 \%$ of cases of rheumatoid arthritis. We have used the SouterStrathclyde total elbow arthroplasty (Stryker Howmedica Osteonics, London, UK) since 1991 and patients have been followed prospectively. We present the clinical outcome and survivorship of arthroplasties performed between 1991 and 1996 with a minimum follow-up of five years.

\section{Patients and Methods}

Between 1991 and 1996, we performed 47 primary total elbow arthroplasties on 38 women and nine men with a mean age at surgery of 61 years for the women and 53 years for the men. The age range was 42 to 87 years. The right to left ratio was 30:17. All patients were evaluated before and after surgery using our own condition-specific outcome measure based on scoring systems available at the time ${ }^{2,3}$ and designed for ease of use. The components of this outcome measure were pain (50 points), function (30 points) and range of movement (20 points) with a maximum aggregate score of 100. The outcome measure and scores for each domain is summarised in Table I.

Patients were seen before the operation, at three and 12 months, and annually thereafter. The last clinical examination for the purpose of our study was undertaken by one of the authors (MK) in a specially designated clinic. The examining author did not have access to the previous data, clinical notes or radiographs at the time of assessment. The pre-operative radiographs, those taken immediately after the operation and those nearest to the time of final clinical review were evaluated.

The pre-operative radiological grading of rheumatoid arthritis of the elbow was done according to the system described by Morrey and Adams. ${ }^{4}$ The immediate post-operative radiographs were evaluated for uniformity of the cement mantle around the implant with special reference to any voids or defects. The radiograph taken at the time of the final clinical review was evaluated for loosening of the implant according to the criteria proposed by Schneeberger, Adams and Morrey. ${ }^{5}$

Eight patients (nine elbows) had died by the time of the final clinical review (mean followup 65 months (36 to 83)) and one patient was lost to follow-up at one year. The last available data for these ten elbows were used for analysis.

The prosthesis. The Souter-Strathclyde total elbow replacement has components which mimic the anatomy of the humero-ulnar joint. The prosthesis is unlinked and partially constrained. Stability is dependent on the accurate 
Table I. Details of the clinical scoring system ${ }^{2,3}$

\begin{tabular}{|c|c|}
\hline Criteria measured & Score \\
\hline None & 50 \\
\hline Slight occasional analgesia & 45 \\
\hline Moderate daily analgesia & 35 \\
\hline Moderate rest/night pain & 15 \\
\hline Severe unremitting & 0 \\
\hline Function & Total 30 \\
\hline No limits & 30 \\
\hline Slight, no restriction $A D L^{*}$ & 25 \\
\hline Unable to lift heavy objects & 20 \\
\hline Moderate restriction ADL & 10 \\
\hline Unable to comb hair & 5 \\
\hline Unable to feed & 0 \\
\hline Movement ( $\left.{ }^{\circ}\right)$ & Total 20 \\
\hline Flexion & Maximum 6 \\
\hline$>125$ & 6 \\
\hline 100 to 125 & 4 \\
\hline 75 to 99 & 2 \\
\hline$<75$ & 0 \\
\hline Extension & Maximum 6 \\
\hline$<15$ & 6 \\
\hline 15 to 45 & 4 \\
\hline 46 to 90 & 2 \\
\hline$>90$ & 0 \\
\hline Pronation & Maximum 4 \\
\hline$>60$ & 4 \\
\hline 30 to 60 & 3 \\
\hline 15 to 29 & 2 \\
\hline$<15$ & 0 \\
\hline Supination & Maximum 4 \\
\hline$>60$ & 4 \\
\hline 45 to 60 & 3 \\
\hline 15 to 44 & 2 \\
\hline$<15$ & 0 \\
\hline Total & Maximum 100 \\
\hline
\end{tabular}

placement of the components and on the integrity of the adjoining soft tissues. The standard vitallium humeral components are small, medium or large. Humeral components with long stems are available. The polyethylene ulnar components are small and medium. All humeral and ulnar components can be paired.

Operative technique. All the operations were performed by the senior author (ANS). The anaesthetised patient was placed in the lateral position on a vacuum pack with the arm over a support bar and forearm hanging vertical to allow free movement. A tourniquet was used in all patients. The mean duration of surgery by continuous tourniquet time was 127 minutes (90 to 180) and was 155 minutes during the first year of the study. A posterior approach fashioning a tongue of triceps based on the ulna was used and the ulnar nerve routinely identified but not dissected from its bed. The radial head was excised. The distal humerus and proximal ulna were prepared using a high-speed air drill and burr. Care was taken to align the trochlear notch of the prosthesis with the olecranon. Implants of the largest
Table II. Mean changes in the elbow score, ${ }^{2,3}$ its domain and range of movement $\left({ }^{\circ}\right)$ after surgery

\begin{tabular}{|c|c|c|c|c|c|}
\hline & \multirow[b]{2}{*}{ Pre-op } & \multicolumn{2}{|c|}{ 1-year follow-up } & \multicolumn{2}{|c|}{ Final follow-up } \\
\hline & & Score & $\begin{array}{l}p \text { value, pre- } \\
\text { op and } 1 \text { year }\end{array}$ & Score & $\begin{array}{l}\text { p value, pre-op } \\
\text { and final score }\end{array}$ \\
\hline \multicolumn{6}{|l|}{ Elbow score } \\
\hline Overall & 47 & 86 & $<0.001$ & 80 & $<0.001$ \\
\hline Pain & 21 & 49 & $<0.001$ & 47 & $<0.001$ \\
\hline Function & 11 & 21 & $<0.001$ & 19 & $<0.001$ \\
\hline Movement & 15 & 15 & $<0.001$ & 15 & 0.060 \\
\hline \multicolumn{6}{|c|}{ Range of movement $\left({ }^{\circ}\right)$} \\
\hline Flexion & 125 & 141 & $<0.001$ & 136 & $<0.001$ \\
\hline Extension & 45 & 45 & 0.95 & 48 & 0.095 \\
\hline Supination & 63 & 64 & 0.553 & 69 & 0.016 \\
\hline Pronation & 56 & 67 & $<0.001$ & 68 & $<0.001$ \\
\hline
\end{tabular}

possible size were fixed with Palacos cement containing gentamicin (Schering-Plough, Kenilworth, New Jersey) using digital pressure only. Cement restrictors and pressurisation devices were not used. We used 26 large and 21 medium humeral components and 28 medium and 19 small ulnar components. The usual combination was an ulnar component of one size smaller than the humeral component.

During the initial part of the study a posterior plaster slab was applied for five days at $90^{\circ}$ of flexion, but the practice has gradually evolved of using the backslab for two days with the elbow in maximal extension. A custom fibreglass posterior splint with a hole cut over the olecranon was worn for three weeks between sessions of extension with gravity and active flexion, initially under supervision of the physiotherapist and then independently.

Statistical analysis. This was done using XLSTAT Version 7.0 statistical software (Addinsoft, New York, New York). The non-parametric distribution of the variables was established by the Shapiro-Wilk test and the Wilcoxon signedranks test was used to compare the variables. Kaplan-Meier survival analysis was done using different end-points.

\section{Results}

The total score, the breakdown of scores for pain, function and movement and the measured range of movement with $\mathrm{p}$ values are detailed in Table II. The mean follow-up was 82 months (12 to 129). Relief from pain and improvement of function were significant. The difference between the pre-operative and final scores for movement was not significant.

The mean range of elbow flexion before the operation was $45^{\circ}\left(15^{\circ}\right.$ to $\left.85^{\circ}\right)$ to $125^{\circ}\left(94^{\circ}\right.$ to $\left.145^{\circ}\right)$ improving at one year to $45^{\circ}\left(20^{\circ}\right.$ to $\left.80^{\circ}\right)$ to $141^{\circ}\left(110^{\circ}\right.$ to $\left.155^{\circ}\right)$ and finally to $48^{\circ}\left(10^{\circ}\right.$ to $\left.85^{\circ}\right)$ to $136^{\circ}\left(115^{\circ}\right.$ to $\left.155^{\circ}\right)$.

The mean arc of flexion/extension before surgery was $80^{\circ}$ $\left(25^{\circ}\right.$ to $\left.119^{\circ}\right)$. At one year it was $95^{\circ}\left(60^{\circ}\right.$ to $\left.130^{\circ}\right)$ and finally $88^{\circ}\left(55^{\circ}\right.$ to $\left.115^{\circ}\right)$.

The mean pre-operative rotation of the forearm was $63^{\circ}$ of supination $\left(30^{\circ}\right.$ to $\left.90^{\circ}\right)$ and $56^{\circ}\left(0^{\circ}\right.$ to $\left.80^{\circ}\right)$ of pronation, 


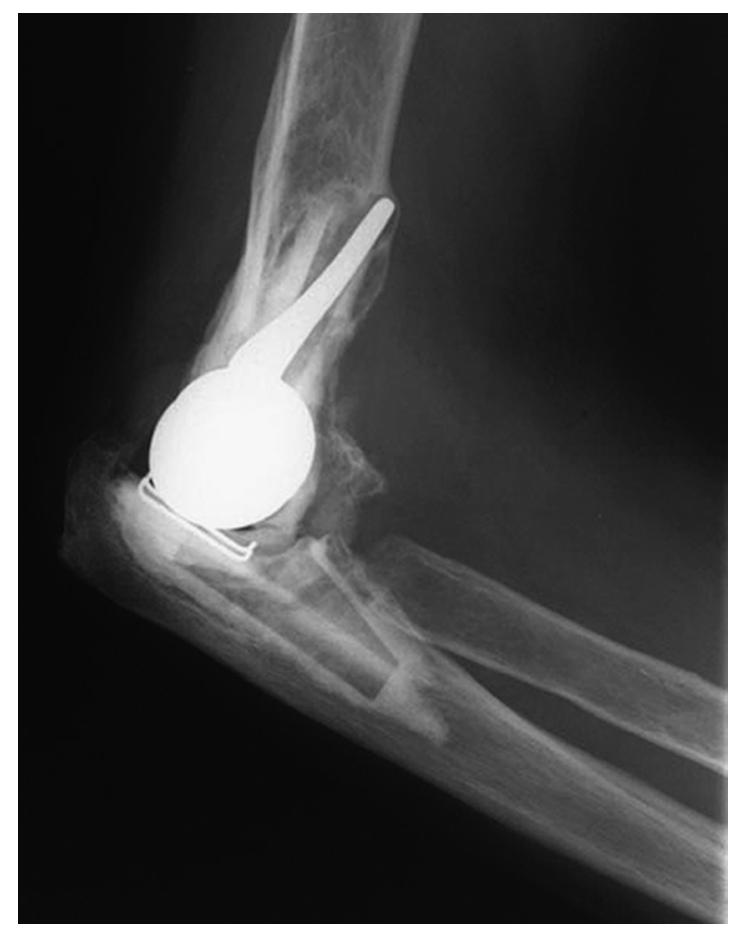

Fig. 1

Post-operative lateral radiograph of the elbow showing loosening associated with late-onset deep infection and early lucency about the ulnar component. The anterior tilting of the humerus is similar to the pattern described for aseptic loosening. Excision arthroplasty was performed since the patient declined revision arthroplasty.

improving slightly at one year to $64^{\circ}\left(18^{\circ}\right.$ to $\left.90^{\circ}\right)$ of supination and $67^{\circ}\left(10^{\circ}\right.$ to $\left.90^{\circ}\right)$ of pronation. The final mean supination was $69^{\circ}\left(31^{\circ}\right.$ to $\left.86^{\circ}\right)$ and pronation $68^{\circ}\left(5^{\circ}\right.$ to $\left.85^{\circ}\right)$.

The mean arc of supination/pronation initially was $120^{\circ}$ $\left(50^{\circ}\right.$ to $\left.160^{\circ}\right)$ which improved to $131^{\circ}\left(28^{\circ}\right.$ to $\left.165^{\circ}\right)$ at 12 months. At the final review it was $136^{\circ}\left(90^{\circ}\right.$ to $\left.145^{\circ}\right)$.

Radiological evaluation. The pre-operative radiographs of 42 patients were available at the time of the final clinical review. The classification of Morrey and Adams ${ }^{4}$ was used to quantify the rheumatoid process. Ten elbows $(23.8 \%)$ were grade II, $25(59.5 \%)$ grade III and seven $(16.6 \%)$ grade IV.

The immediate post-operative radiographs $(n=45)$ were evaluated for the adequacy of cementation and positioning of the prosthesis. The medullary canal was completely filled with cement without any defect in the cement mantle or sign of radiolucency at the cement-bone interface in 43 humeral components and in 44 ulnar components. A radiolucent line at the cement-bone interface was seen in two humeral and one ulnar component. In all three cases this radiolucent line was less than $1 \mathrm{~mm}$ thick and involved less than $50 \%$ of the interface.

At the final review radiographs were evaluated using the criteria outlined by Schneeberger et al. ${ }^{5}$ Type I loosening was seen in 35 humeri and 37 ulnae, type II in three humeri and one ulna and type III in one humerus and two ulnae. The patient with type III humeral loosening had a pain score of 15 at the time of the final review, but revision was declined. Gross loosening of the implant (type IV) was observed in four humeri and three ulnae. Two of these cases were associated with late-onset deep infection (Fig. 1). One patient had aseptic loosening and associated fracture in the supracondylar region. A wide localised area of radiolucency was seen around the humeral implant in one patient who was asymptomatic. Open biopsy showed this to be metastatic disease from the lung. Four radiographs were not available for review. Three patients had died and one was lost to follow-up.

Survivorship. Kaplan-Meier survivor analysis was done using XLSTAT Version 7.0 statistical software using the following end-points: 1 ) removal of implants for aseptic loosening (Fig. 2); 2) removal of implants for any reason (Fig. 3); and 3) a pain score of 35 or less at the time of the final review (Fig. 4). Four elbows have been removed to date, one for aseptic loosening and three for deep infection. The cumulative rate of survival for implants removed for aseptic loosening was $97.7 \%$ with a mean survival time of 123 months (SD 2.8; 95\% confidence interval (CI) 112 to 118). The cumulative rate of survival for implants removed for any reason was $75.0 \%$ with a mean survival time of 114 months (SD 2.83; 95\% CI 109 to 120 ) and that using the pain score as a secondary outcome measure $95 \%$ with a mean survival time of 116 months (SD 4.16; 95\% CI 108 to 124). The cumulative rate of survival using radiological loosening (grades III and IV) as a secondary outcome measure was $88 \%$ with a mean survival time of 113 months (SD 3.22; $95 \%$ CI 106 to 119 ).

Complications (Table III). Early deep infection by Staphylococcus epidermidis was encountered in one patient after dislocation and wound dehiscence requiring removal of the implants within four weeks of operation. Re-implantation surgery was declined.

Late-onset deep infection requiring removal of the implant occurred in two patients at 83 and 85 months. Both patients were culture-negative and had been prescribed antibiotics before attending hospital. One patient presented with a discharging wound after surgery had been performed on the olecranon bursa at another hospital. The implants were removed and the elbow was stabilised using an Ilizarov frame, but the patient died shortly after from respiratory disease. Two-stage revision was performed in the other patient who presented with pain, swelling and discharge from the elbow.

Revision for aseptic loosening was performed at 106 months in one patient who presented with pain of a few weeks' duration after minor trauma. Radiographs showed a loose implant and a supracondylar fracture with formation of callus. The elbow was revised using a long-stemmed component. For the patient with metastatic disease revision to a long-stemmed component was done to prevent fracture and this has not been classified as failure of the implant. 


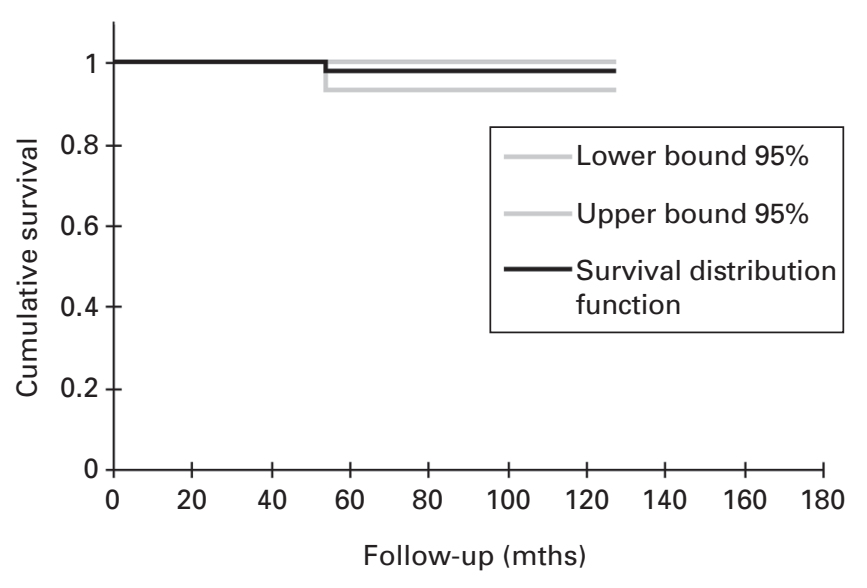

Fig. 2

Survival of the Souter-Strathclyde elbow replacement using revision due to aseptic loosening as the definition of failure with $95 \%$ confidence intervals.

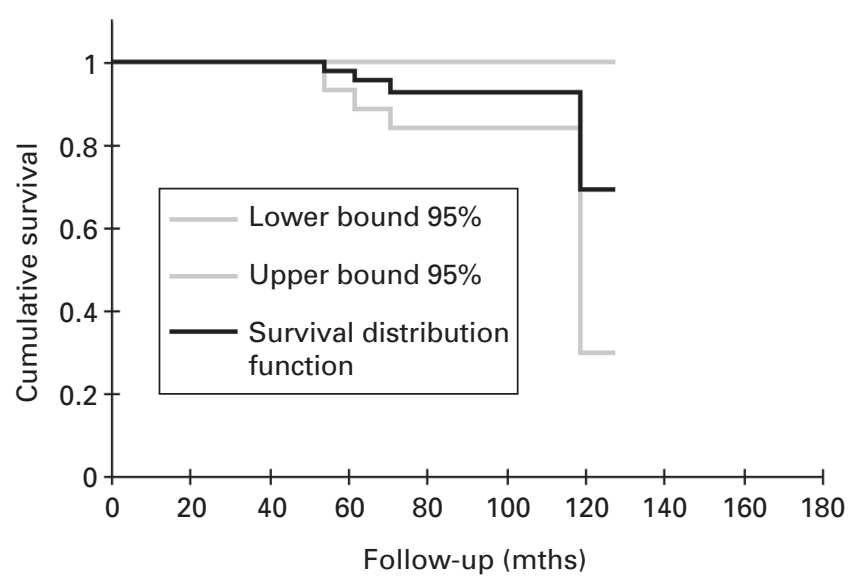

Fig. 3

Survival of the Souter-Strathclyde elbow replacement using removal of the elbow for any cause as the definition of failure with $95 \%$ confidence intervals.

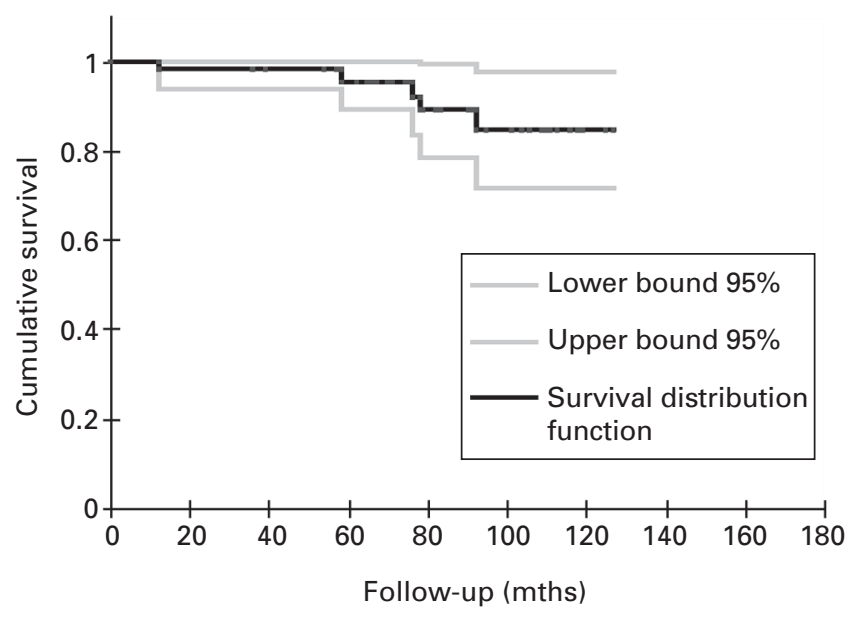

Fig. 4

Survival of the Souter-Strathclyde elbow replacement using pain as a secondary outcome measure with $95 \%$ confidence intervals.
Table III. Complications seen in 47 total elbow arthroplasties

\begin{tabular}{llc}
\hline Complication & Number & $\%$ \\
\hline Early-onset deep infection & 1 & 2.1 \\
Late-onset deep infection & 2 & 4.2 \\
Aseptic loosening requiring revision & 1 & 2.1 \\
Superficial infection & 1 & 2.1 \\
Temporary dysfunction of the ulnar nerve & 6 & 12.7 \\
Permanent sensory impairment of the ulnar nerve & 1 & 2.1 \\
Minor intra-operative fracture & 1 & 2.1 \\
Dislocation & 3 & 6.3
\end{tabular}

Six patients had temporary symptoms in the distribution of the ulnar nerve after the operation but were asymptomatic at the final review. One patient had permanent dysaesthesia in the distribution of the ulnar nerve.

A minor fracture of the lateral condyle of the humerus in one patient required no specific intervention. There were three dislocations in the early post-operative period because of rupture of the triceps mechanism, which was successfully repaired in all cases.

\section{Discussion}

Total elbow arthroplasty is well established in the treatment of patients with rheumatoid arthritis of the elbow. Unlinked prostheses such as the Souter-Strathclyde ${ }^{6,7}$ or Kudo $^{8}$ have been advocated for elbows with minimum bone destruction and preserved soft tissues while a linked prosthesis such as the Coonrad-Morrey may be preferred in elbows with destruction of bone and gross instability. ${ }^{9}$

In our study, the severity of the disease by grading had no influence on the duration of the operation. We encountered no complications related to the tourniquet which was adjusted to no more than $50 \mathrm{mmHg}$ above the systolic pressure. The medial collateral ligament was not specifically preserved. Great care was taken to ensure that, in the lateral position with the upper arm resting on the support bar and the forearm hanging vertical, the trial components were in balance and appropriately aligned. Although $16 \%$ of the elbows in our series had advanced destruction of the elbow, standard implants were used in all cases. In the presence of gross erosion, insertion of the ulnar component required care in order to avoid fracture.

In the simple outcome measure which we used pain is weighted heavily and the range of movement lightly which we acknowledge may introduce bias in the case of severe rheumatoid disease. There were significant increases in the score for pain and function, but not for movement. The reduction in the overall score between follow-up at one year and the final clinical review may be attributable to the cases requiring revision or removal of the implant.

Gains in flexion of $14^{\circ}$ and in pronation of $10^{\circ}$ were statistically significant, but there was no significant change in extension or supination. Flexion of $136^{\circ}$ is similar to other published results for the Souter-Strathclyde, ${ }^{6,7} \mathrm{Kudo}^{8}$ and Coonrad-Morrey ${ }^{9}$ prostheses. Less fixed flexion has been reported for the linked Coonrad-Morrey ${ }^{9}$ implant. 
Table IV. Size of the humeral and ulnar components used in this series $(n=47)$ and by Rozing $(n=66)^{13}$

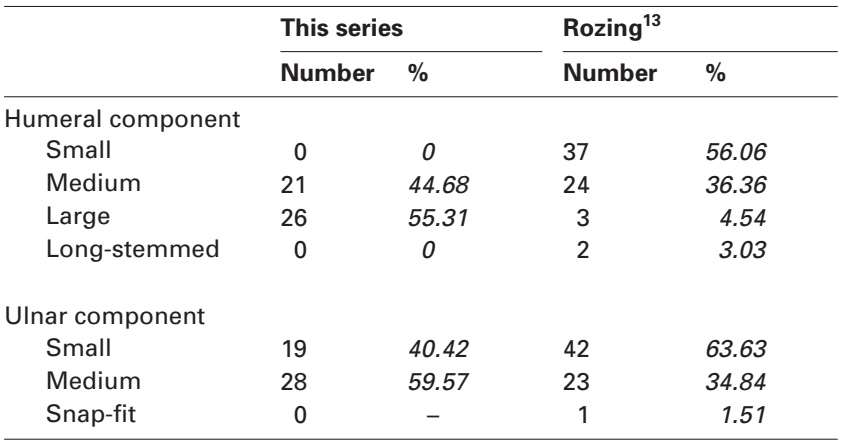

Adequate cementation with a uniform mantle around the prosthesis is associated with a decreased incidence of aseptic loosening and increased survivorship of hip replacements. ${ }^{10,11}$ A uniform mantle around both humeral and ulnar components was seen in all post-operative radiographs with minor radiolucent lines around only three components.

One case of aseptic loosening was successfully revised. One humeral and two ulnar components had grade-III loosening according to Schneeberger et $\mathrm{al}^{5}$ (radiolucency around the entire components) without related symptoms. Grade IV (gross loosening) of the component seen in two humeri and two ulnae was associated with late-onset deep infection. The mode of failure of the humeral components was by anterior tilting as described by Trail et al. ${ }^{7}$ One of the three cases complicated by infection occurred early in the series and was associated with malalignment of the components causing dislocation and wound dehiscence requiring early removal of the implant. The other two infections were of late onset, presumably haematogenous.

For the Souter-Strathclyde prosthesis, van der Lugt et al ${ }^{6}$ reported survival of $77 \%$ at ten years, the end-point being revision with or without re-insertion of a new implant. Trail et $\mathrm{al}^{7}$ has quoted a survivorship of $87 \%$ at 12 years. Survivorship for the Kudo ${ }^{8}$ and Coonrad-Morrey ${ }^{9}$ implants are, respectively, $90 \%$ at 16 years and $92 \%$ at ten years. Our rate of survival is $97 \%$ with a mean survival time of ten years if revision for aseptic loosening is taken as the endpoint. If removal of implants for whatever reason, including the three deep infections, is taken as the end-point, the survival rate falls to $75 \%$ at nine years.

In principle, the largest possible implants were used (Table IV) based on the suggestion of both the designer ${ }^{1,12}$ and Rozing ${ }^{13}$ that the larger the humeral component is the less is the risk of loosening because of optimisation of epicondylar fixation. We believe that, despite the requirement to remove more bone, the use of larger implants and the ensuring of a uniform cement mantle are important to reducing the rate and extent of loosening although this is not proven.

Closed dislocation was dealt with in three elbows by repair of triceps. The rate of dysaesthesia of the ulnar nerve was low. In our series the standard practice was to identify, but not to mobilise, the ulnar nerve thus preserving the neighbouring soft-tissue envelope and blood supply. Overall, the rate of complications was $34 \%$. Half of these patients had no significant consequences.

We conclude that the Souter-Strathclyde total elbow replacement affords relief from pain and functional improvement in rheumatoid patients with relatively low rates of major complications, loosening and requirement for revision. However, the procedure requires extensive resection of bone, preservation of soft tissues and meticulous alignment of the implants.

No benefits in any form have been received from a commercial party related directly or indirectly to the subject of this article.

\section{References}

1. Souter WA. Surgery for rheumatoid arthritis: upper limb surgery of the elbow. Curr Orthop 1989;3:9-13

2. Ewald FC. Total elbow replacement. Orthop Clin North Am 1975;6:685-96.

3. Inglis AE, Pellicci PM. Total elbow replacement. J Bone Joint Surg [Am] 1980; 62-A:1252-8.

4. Morrey BF, Adams RA. Semiconstrained arthroplasty for the treatment of rheumatoid arthritis of the elbow. J Bone Joint Surg [Am] 1992;74-A:479-90.

5. Schneeberger AG, Adams R, Morrey BF. Semiconstrained total elbow replacement for the treatment of post-traumatic osteoarthrosis. J Bone Joint Surg [Am]1997; 79-A:1211-22

6. van der Lugt JC, Geskus RB, Rozing PM. Primary Souter-Strathclyde total elbow prosthesis in rheumatoid arthritis. J Bone Joint Surg [Am] 2004;86-A:465-73.

7. Trail IA, Nuttall D, Stanley JK. Survivorship and radiographic analysis of the standard Souter-Strathclyde total elbow arthroplasty. J Bone Joint Surg [Br] 1999;81-B: 80-4.

8. Tanaka N, Kudo H, Iwano K, et al. Kudo total elbow arthroplasty in patients with rheumatoid arthritis: a long-term follow-up study. J Bone Joint Surg [Am] 2001;83-A: 1506-13.

9. Gill DRJ, Morrey BF. The Coonrad-Morrey total elbow arthroplasty in patients who have rheumatoid arthritis: a ten to fifteen-year follow-up study. J Bone Joint Surg [Am] 1998;80-A:1327-35.

10. Barrack RL, Mulroy RD Jr, Harris WH. Improved cementing techniques and femoral component loosening in young patients with hip arthroplasty: a 12-year radiographic review. J Bone Joint Surg [Br] 1992;74-B:385-9.

11. Mulroy WF, Estok DM, Harris WH. Total hip arthroplasty with use of so called second-generation cementing techniques: a fifteen-year average follow-up study. J Bone Joint Surg [Am] 1995;77-A:1845-52.

12. Stryker Howmedica Ostenics. Souter-Strathclyde total elbow arthroplasty: surgical treatment. 1999.

13. Rozing P. Souter-Strathclyde total elbow arthroplasty. J Bone Joint Surg [Br] 2000; 82-B:1129-34. 